\title{
Role of miR-589-3p in human lumbar disc degeneration and its potential mechanism
}

\author{
AIQING LU, ZHIRONG WANG and SUCHUN WANG

\begin{abstract}
Department of Orthopaedics, Zhangjiagang Hospital of Traditional Chinese Medicine Affiliated to
\end{abstract} \\ Nanjing University of Chinese Medicine, Zhangjiagang, Jiangsu 215600, P.R. China
}

Received August 8, 2017; Accepted November 15, 2017

DOI: $10.3892 /$ etm.2017.5593

\begin{abstract}
The present study aimed to investigate the role of miR-589-3p in lumbar disc degeneration (LDD) and to explore the underlying mechanisms. Nucleus pulposus (NP) cells were stimulated with lipopolysaccharide (LPS) to simulate an in vitro model of intervertebral disc degeneration. Reverse transcription-quantitative polymerase chain reaction (RT-qPCR) was used to detect the expression level of microRNA (miR)-589-3p in the NP cells, and the results demonstrated that the increased expression of miR-589-3p in LPS stimulated NP cells compared with the control. To further investigate the role of miR-589-3p in LDD, a human NP cell line with high/low miR-589-3p expression was generated using miR-589-3p mimics/inhibitors. In addition, a human NP cell inflammation model was conducted by LPS $(10 \mu \mathrm{M})$ treatment. Western blot analysis and RT-qPCR were performed for detection of associated genes and proteins. Protein levels of pro-inflammatory factors, including tumor necrosis factor- $\alpha$ (TNF- $\alpha$ ), interleukin (IL)- $1 \beta$ and IL- 6 were evaluated by ELISA. Flow cytometry was used for cell apoptosis determination. Furthermore, Targetscan was used to predict potential targets of miR-589-3p, and a dual luciferase reporter assay was used to verify the prediction. The findings verified that miR-589-3p was significantly upregulated in LDD. In vitro, miR-589-3p mimics/inhibitors significantly increased/reduced the production of TNF- $\alpha$, IL- $1 \beta$ and IL- 6 in LPS stimulated NP cells. Furthermore, miR-589-3p mimics/inhibitors significantly promoted/inhibited LPS stimulated NP cell apoptosis. MiR-589-3p mimics/inhibitors significantly repressed/enhanced type II collagen and aggrecan expression in LPS stimulated NP cells. In addition, it was demonstrated that mothers against decapentaplegic homolog (Smad) 4 was a direct target gene of miR-589-3p, and was negatively regulated by miR-589-3p in NP
\end{abstract}

Correspondence to: Dr Suchun Wang, Department of Orthopaedics, Zhangjiagang Hospital of Traditional Chinese Medicine Affiliated to Nanjing University of Chinese Medicine, 77 Chang'an South Road, Zhangjiagang, Jiangsu 215600, P.R. China E-mail: wangsuchun08@163.com

Key words: lumbar disc degeneration, miR-589-3p, Smad4, pro-inflammatory factors, apoptosis, type II collagen cells. In conclusion, miR-589-3p may function as a promoter in LDD development via the regulation of Smad4.

\section{Introduction}

Lumbar disc degeneration (LDD) is the premise and pathological basis of the degenerative lumbar diseases (1). As a common clinical disease, related survey shows that about $80 \%$ of the LDD patients will have different degree of back and leg pain symptoms, and it may affect their daily work and life quality when the pain is relatively serious. At present, the mechanism of intervertebral disc degeneration (IDD) has not been fully understood. Various factors, including lifestyle, age, genetic predisposition, etc, can induce IDD (2,3). Evidence have indicated that a variety of cellular events, ranging from matrix synthesis to cytokine expression, are participated in the development of human IDD (4). A growing number of studies suggested that nucleus pulposus (NP) cells, which can produce type II collagen, aggrecan and other components of the extracellular matrix (ECM), play a major role in maintaining the integrity of intervertebral discs (IVDs) $(5,6)$. Moreover, during the progression of IDD, excessive apoptosis of IVD cells and excessive degradation of ECM are observed. In addition, pro-inflammatory cytokines, such as tumor necrosis factor- $\alpha$ (TNF- $\alpha$ ), interleukin (IL)- $1 \beta$ and IL-6, also play critical roles in IDD.

MicroRNAs (miRNAs), a class of short non-coding RNAs (20-22 nucleotides), play critical roles in post-transcriptional regulation of target gene expression $(7,8)$. Increasing studies have reported that miRNAs play an important role in a series of pathological processes, such as neurodegenerative, inflammatory and degenerative disorders $(9,10)$. In recent years, a number of studies have revealed that miRNAs serve a major role in degenerative disc diseases, such as IDD (11-15).

miR-589 has been reported over-expressed in IDD (16). However, the expression and role of miR-589-3p in LDD remains largely unclear. Therefore, the present study aimed to investigate the expression and role of miR-589-3p in LDD, as well as the underlying molecular mechanisms.

\section{Materials and methods}

Materials. Lipopolysaccharide (LPS) was obtained from Sigma-Aldrich (Merck, Darmstadt, Germany); The cell transfection kit was purchased from Santa Cruz Biotechnology, Inc. 
(Dallas, TX, USA); ELISA kits for TNF- $\alpha$, IL-1 $\beta$ and IL-6 level detection were purchased from Elabscience Biotechnology (Wuhan, China); The primary antibodies ( $\beta$-actin, Smad4, type II collagen, aggrecan) and the secondary antibody were obtained from Cell Signaling Technology (Danvers, MA, USA); All other chemicals and reagents were supplied by Sinopharm Chemical Reagent (Shanghai, China).

Cell culture. The primary human NP cells were obtained from the ScienCell Research Laboratories (Santiago, CA, USA). Cells were grown in DMEM (Gibco; Thermo Fisher Scientific, Inc., Waltham, MA, USA) containing $10 \%$ fetal bovine serum (FBS, Gibco; Thermo Fisher Scientific, Inc.), $1 \%$ streptomycin-penicillin solution, and $1 \%$ L-glutamine (Gibco; Thermo Fisher Scientific, Inc.). The cells were maintained in a $5 \% \mathrm{CO}_{2}$ incubator at $37^{\circ} \mathrm{C}$.

Cell transfection. Human NP cells were seeded in 6-well plates $\left(5 \times 10^{4}\right.$ cells/well) the day before cell transfection. miR-589-3p mimic (50 nM), miR-589-3p inhibitor (100 nM) or its negative control (NC) (GenScript, Piscataway, NJ, USA) were transfected in to NP cells by using Lipofectamine 2000 (Invitrogen; Thermo Fisher Scientific, Inc.) according to the manufacturer's instructions.

LPS treatment. $24 \mathrm{~h}$ after the cell transfection, cells were treated with $10 \mathrm{ng} / \mathrm{ml} \mathrm{LPS} \mathrm{for} 24 \mathrm{~h}$ and incubated in a $5 \% \mathrm{CO}_{2}$ incubator at $37^{\circ} \mathrm{C} .24 \mathrm{~h}$ after treatment, the supernatants were harvested by centrifugation $\left(1,000 \mathrm{x} \mathrm{g} ; 4^{\circ} \mathrm{C} ; 15 \mathrm{~min}\right)$. Cells were divided into following groups: Control group (Con): Cells without any treatment; LPS treatment group (LPS): Cells treated with LPS; NC/LPS group: Cells transfected with negative control and then stimulated with LPS; miR-589-3p/LPS group: Cells transfected with miR-589-3p mimics and then stimulated with LPS; inhibitor/LPS group: Cells transfected with miR-589-3p inhibitor and then stimulated with LPS. Then cells were collected for following analysis.

Reverse transcription-quantitative polymerase chain reaction $(R T-q P C R)$. Total RNA from NP cells was extracted by using TRIzol reagent (Takara Bio, Inc., Otsu, Japan) according to the manufacturer's protocol. cDNAs were generated by using the miScript Reverse Transcription kit (Qiagen GmbH, Hilden, Germany) according to the instructions provided by the manufacturer. Subsequently, qPCR was performed using Maxima SYBR-Green/ROX qPCR Master mix (Thermo Fisher Scientific, Inc.) according to the manufacturer's protocol. Primer sequences used were obtained from Genscript (Nanjing, China). U6 or GAPDH was served as the internal control of miRNA or mRNA expression. The $2^{-\Delta \Delta \mathrm{Cq}}$ method was used to calculate the relative expression values (17). Every experiment was repeated at least three times.

Western blot analysis. After certain treatment, NP cells were collected and total cell proteins were extracted by using RIPA buffer (Cell Signaling Technology, Inc., Danvers, MA, USA). Then, bicinchoninic acid protein assay was used to detect the concentration of proteins. Equal amount of protein samples were resolved on $10 \%$ SDS-PAGE gels, and then transferred onto the PVDF membranes (EMD Millipore, Billerica, MA,
USA). The membranes were then blocked with 5\% skim milk, incubated with the primary antibodies (overnight at $4^{\circ} \mathrm{C}$ ), and then incubated with a secondary antibody (room temperature for $2 \mathrm{~h}$ ). Finally, the Chemiluminescent ECL reagent (EMD Millipore) was performed for protein blots visualization.

ELISA assay. $24 \mathrm{~h}$ after cell transfection, NP cells were treated with $10 \mathrm{ng} / \mathrm{ml}$ LPS. Then, the supernatants were harvested for the detection of proinflammatory factors (TNF- $\alpha$, IL-1 $1 \beta$ and IL-6). To determined the level of TNF- $\alpha$, IL- $1 \beta$ and IL-6 of different groups, ELISA assay was applied in line with the manufacturer's instructions of each kit. Each experiment was repeated at least three times.

Flow cytometry assay. Annexin V/propidium iodide double staining method was performed to analyze cell apoptosis. $24 \mathrm{~h}$ after cell transfection, the cells were labeled with Annexin V-FITC and propidium iodide (PI) for cell apoptotic rate detection following the manufacturer's instructions. Flow cytometry (BD Biosciences, Franklin Lakes, NJ, USA) was performed to analyze the cells. Each experiment was independently repeated at least for three times.

Dual luciferase reporter assay. TargetScan was carried out to predict the potential targets of miR-589-3p. To verify whether miR-589-3p directly targets the 3'UTRs of SMAD4, the vectors named SMAD4-3'UTR-WT and SMAD4-3'UTR-MUT with wild-type and mutated 3'UTR of SMAD4 mRNA were constructed. NP cells were co-transfected with SMAD4-3'UTR-WT or SMAD4-3'UTR-MUT and miR-589-3p or its negative control (hsamiR-NC) vector by using Lipofectamine ${ }^{\circledR} 2000$ following the manufacturer's instructions. $48 \mathrm{~h}$ after cell transfection, the Dual-Luciferase Reporter Assay kit (Promega Corporation, Madison, WI, USA) was used to assess the luciferase activity in line with the instructions provided by the manufacturer.

Statistical analysis. Data are presented as the mean \pm standard deviation. Comparisons between groups were analyzed by the Student's t-test and one-way ANOVA. $\mathrm{P}<0.05$ was considered to indicate a statistically significant difference.

\section{Results}

Upregulation of miR-589-3p in LPS stimulated NP cells. To determine the expression level of miR-589-3p in LDD, LDD cell model was generated using LPS in the present study. As shown in Fig. 1A, after stimulated with LPS, the miR-589-3p expression level in NP cells significantly increased compared with the control groups. The data suggested that miR-589-3p was upregulated in LPS stimulated NP cells, indicating that miR-589-3p may be involved in the progression of LDD.

To investigate the role of miR-589-3p in LDD, miR-589-3p was upregulated or downregulated in NP cells by transfection with miR-589-3p mimic, miR-589-3p inhibitor or its negative control. Subsequently, the cells were treated with LPS. $24 \mathrm{~h}$ after the treatment, miR-589-3p expression was determined by RT-qPCR. The results suggested that miR-589-3p 

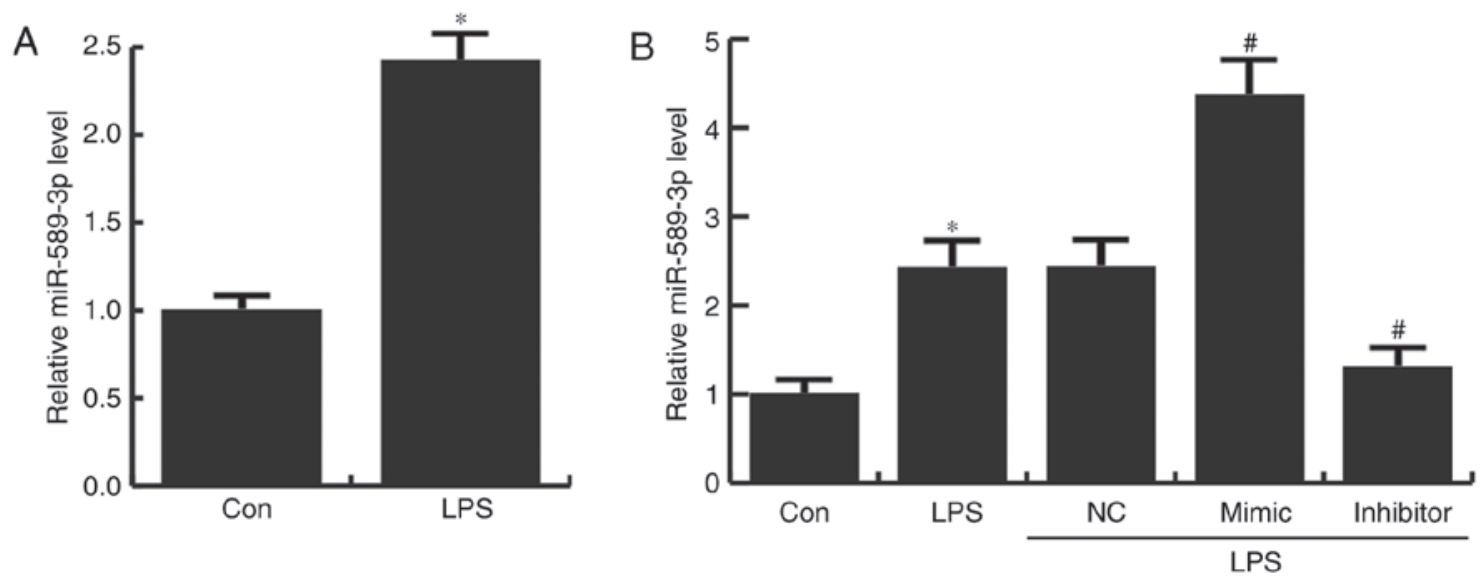

Figure 1. miR-589-3p expression detection. miR-589-3p expression level was measured by RT-qPCR, and data were normalized to U6 expression. (A) miR-589-3p expression level in LPS stimulated NP cells; (B) miR-589-3p expression level in NP cells from different groups. Con, normal NP cells; LPS, NP cells stimulated with LPS; NC, NP cells transfected with NC oligonucleotides and stimulated with LPS; mimic, NP cells transfected with miR-589-3p mimic $(50 \mathrm{nM})$ and stimulated with LPS; inhibitor, NP cells transfected with miR-589-3p inhibitor (100 nM) and stimulated with LPS. "P<0.01 vs. Con; ${ }^{\text {"}} \mathrm{P}<0.01$ vs. LPS. Experiments were performed in triplicate.
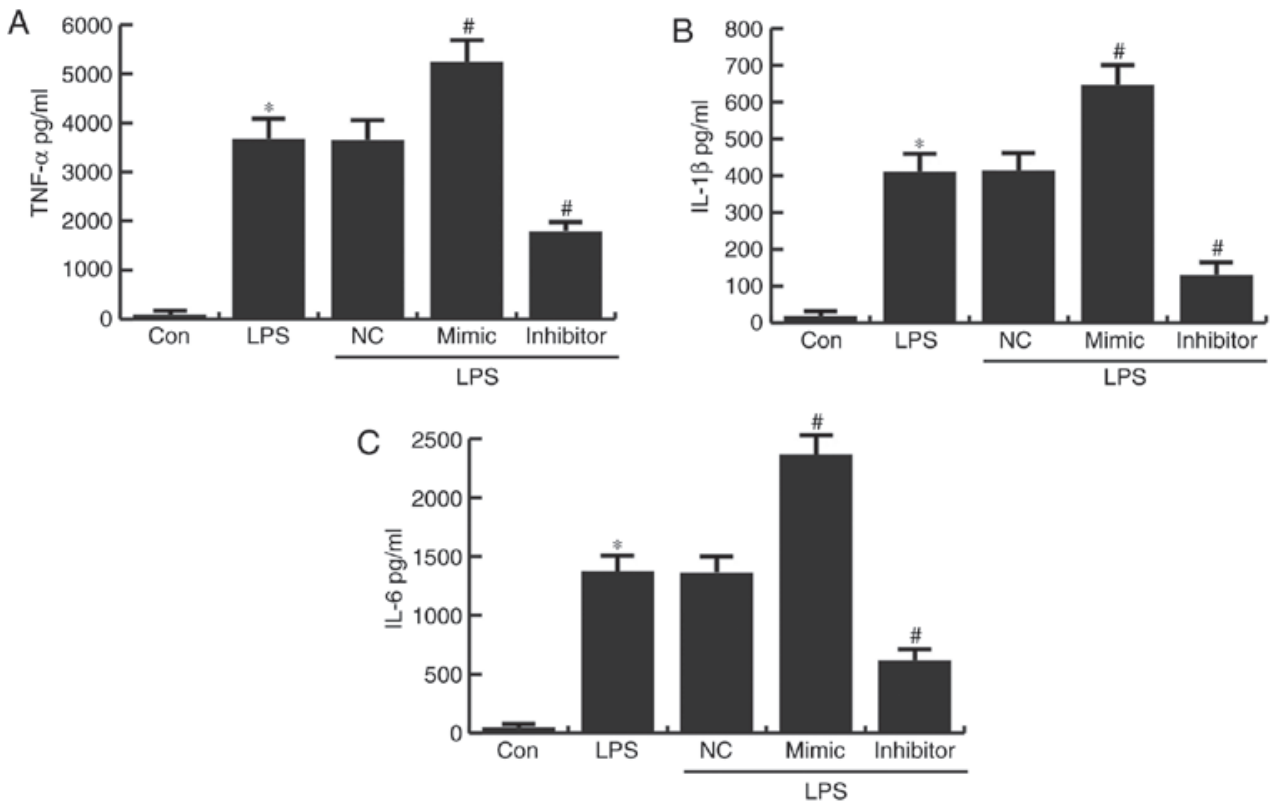

Figure 2. Expression level of cellular supernatant TNF- $\alpha$, IL-1 $\beta$ and IL-6. ELISA assay was performed to detect the expression level of cellular supernatant (A) TNF- $\alpha$, (B) IL-1 $\beta$ and (C) IL-6. Con, normal NP cells; LPS, NP cells stimulated with LPS; NC, NP cells transfected with NC oligonucleotides and stimulated with LPS; mimic, NP cells transfected with miR-589-3p mimic (50 nM) and stimulated with LPS; inhibitor, NP cells transfected with miR-589-3p inhibitor $(100 \mathrm{nM})$ and stimulated with LPS. ${ }^{*} \mathrm{P}<0.01 \mathrm{vs.} \mathrm{Con;}{ }^{*} \mathrm{P}<0.01$ vs. LPS. Experiments were performed in triplicate.

mimic/miR-589-3p inhibitor significantly increased/decreased the expression of miR-589-3p (Fig. 1B).

miR-589-3p inhibition decreased pro-inflammatory cytokines levels in LPS-stimulated NP cells. $24 \mathrm{~h}$ after treatment, the levels of TNF- $\alpha$, IL-1 $\beta$ and IL- 6 were detected by ELISA. We found LPS notably increased the level of TNF- $\alpha$, IL-1 $\beta$ and IL-6. We also found that compared with the LPS stimulated only group, miR-589-3p mimics significantly increased the expression level of TNF- $\alpha$, IL-1 $\beta$ and IL-6. In contrast, miR-589-3p inhibitor markedly inhibited the production of TNF- $\alpha$, IL-1 $\beta$ and IL-6 (Fig. 2A-C). The data indicated that miR-589-3p downregulation significantly prevented the proinflammatory cytokine expression in LPS treated NP cells.
miR-589-3p inhibition decreased LPS induced NP cell apoptosis. The effect of miR-589-3p on NP cell apoptosis was determined in our present study. The results suggested that compared with the control group, LPS treatment significantly enhanced NP cell apoptosis. Compared with the LPS stimulated only group, miR-589-3p mimics significantly promoted the NP cell apoptosis, while miR-589-3p inhibitor markedly inhibited NP cell apoptosis (Fig. 3). The data indicated that miR-589-3p downregulation significantly prevented cell apoptosis in LPS treated NP cells.

miR-589-3p inhibition enhanced type II collagen, aggrecan expression. Effect of miR-589-3p on type II collagen (Col II), aggrecan expression in NP cells was also 

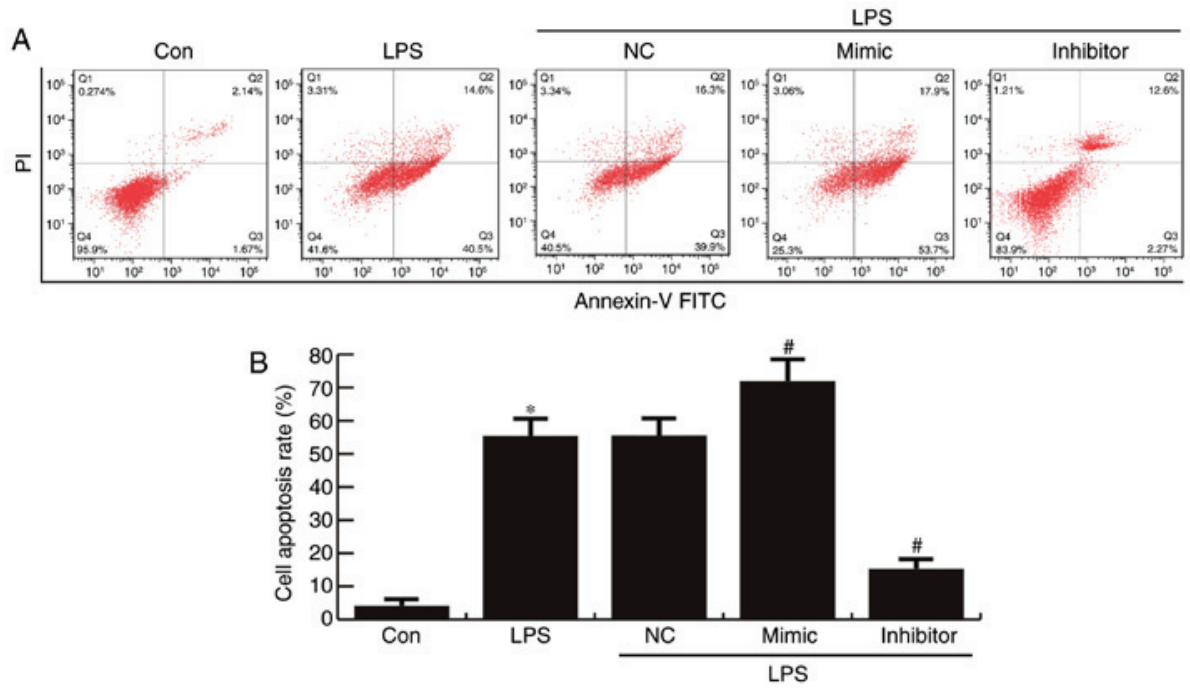

Figure 3. Effect of miR-589-3p on NP cell apoptosis. After specific treatment, NP cell apoptosis was analyzed by FCM. (A) Representative image and (B) quantification. Q1, dead cells; Q2, late apoptosis cells; Q3, early apoptosis cells; Q4, surviving cells. Cell apoptosis=Q2+Q3. Con, normal NP cells; LPS, NP cells stimulated with LPS; NC, NP cells transfected with NC oligonucleotides and stimulated with LPS; mimic, NP cells transfected with miR-589-3p mimic $(50 \mathrm{nM})$ and stimulated with LPS; inhibitor, NP cells transfected with miR-589-3p inhibitor $(100 \mathrm{nM})$ and stimulated with LPS. "P<0.01 vs. Con; ${ }^{\#} \mathrm{P}<0.01$ vs. LPS. Experiments were performed in triplicate.
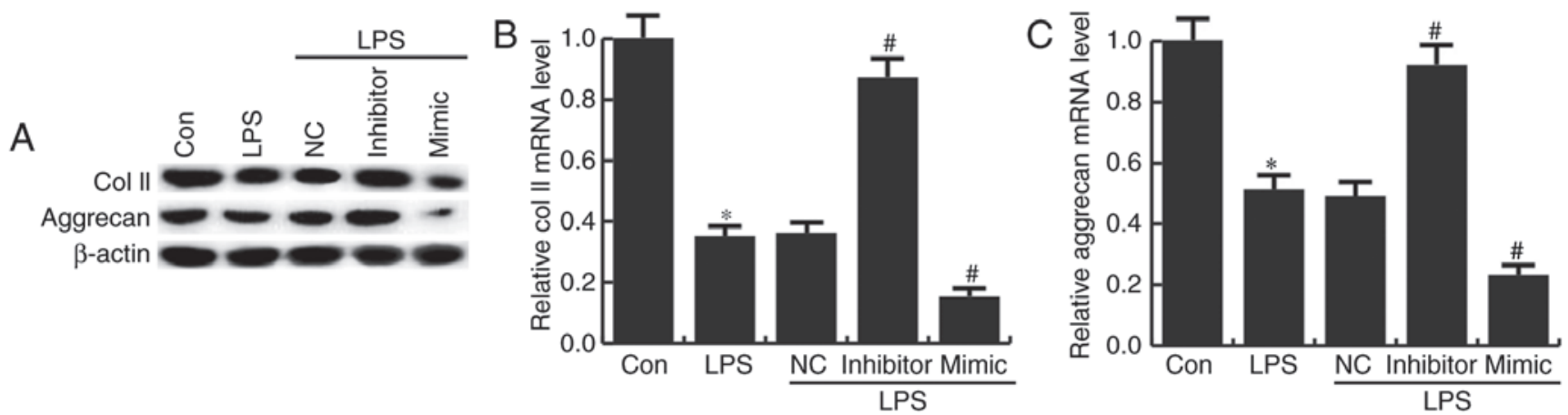

Figure 4. Effect of miR-589-3p on type II collagen, aggrecan expression in NP cells. (A) Protein level of type II collagen and aggrecan was detected by western blotting; (B and C) mRNA level of type II collagen and aggrecan was detected by RT-qPCR. Con, normal NP cells; LPS, NP cells stimulated with LPS; NC, NP cells transfected with NC oligonucleotides and stimulated with LPS; mimic, NP cells transfected with miR-589-3p mimic (50 nM) and stimulated with LPS; inhibitor, NP cells transfected with miR-589-3p inhibitor (100 nM) and stimulated with LPS. "P<0.01 vs. Con; ${ }^{~} \mathrm{P}<0.01$ vs. LPS. Experiments were performed in triplicate.

investigated in the present study. The results indicated that the level of type II collagen and aggrecan was significantly lower in LPS treated NP cells than that in the control NP cells. Compared with the LPS stimulated only group, miR-589-3p mimics significantly inhibited type II collagen and aggrecan expression in NP cells, while miR-589-3p inhibitor markedly enhanced type II collagen and aggrecan expression (Fig. 4).

miR-589-3p targets Smad4. To investigate how miR-589-3p function its role in NP cells, we first predicted the potential targets of miR-589-3p using TargetScan (Fig. 5A), and Dual luciferase reporter Assay was applied to confirm our prediction. The results showed that miR-589-3p effectively reduced luciferase activity in NP cells transfected with the WT 3'UTR of SMAD4 compared to the miR-Con groups, while the MUT SMAD4 3'UTR eliminated the inhibition by miR-589-3p (Fig. 5B). This results indicated that miR-589-3p targets Smad4.
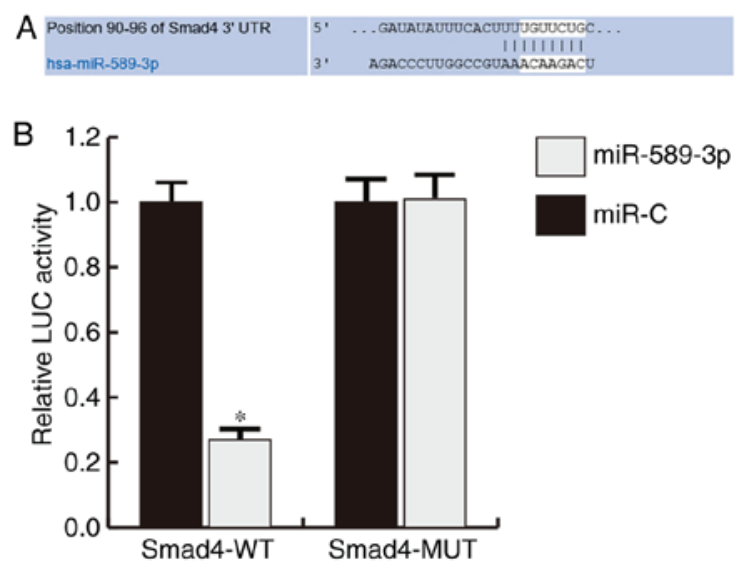

Figure 5. Smad4 is a target gene of miR-589-3p. (A) Interaction between miR-589-3p and 3'UTR of Smad4 was predicted using TargetScan; (B) Luciferase activity of a reporter containing a wild-type Smad4 3'UTR or a mutant Smad4 3'UTR are shown in the bar graph (" $\mathrm{P}<0.01$ vs. control). Here, 'Smad4 Mut 3'UTR' indicates the Smad4 3'UTR with a mutation in the miR-589-3p binding site. UTR, untranslated region. 

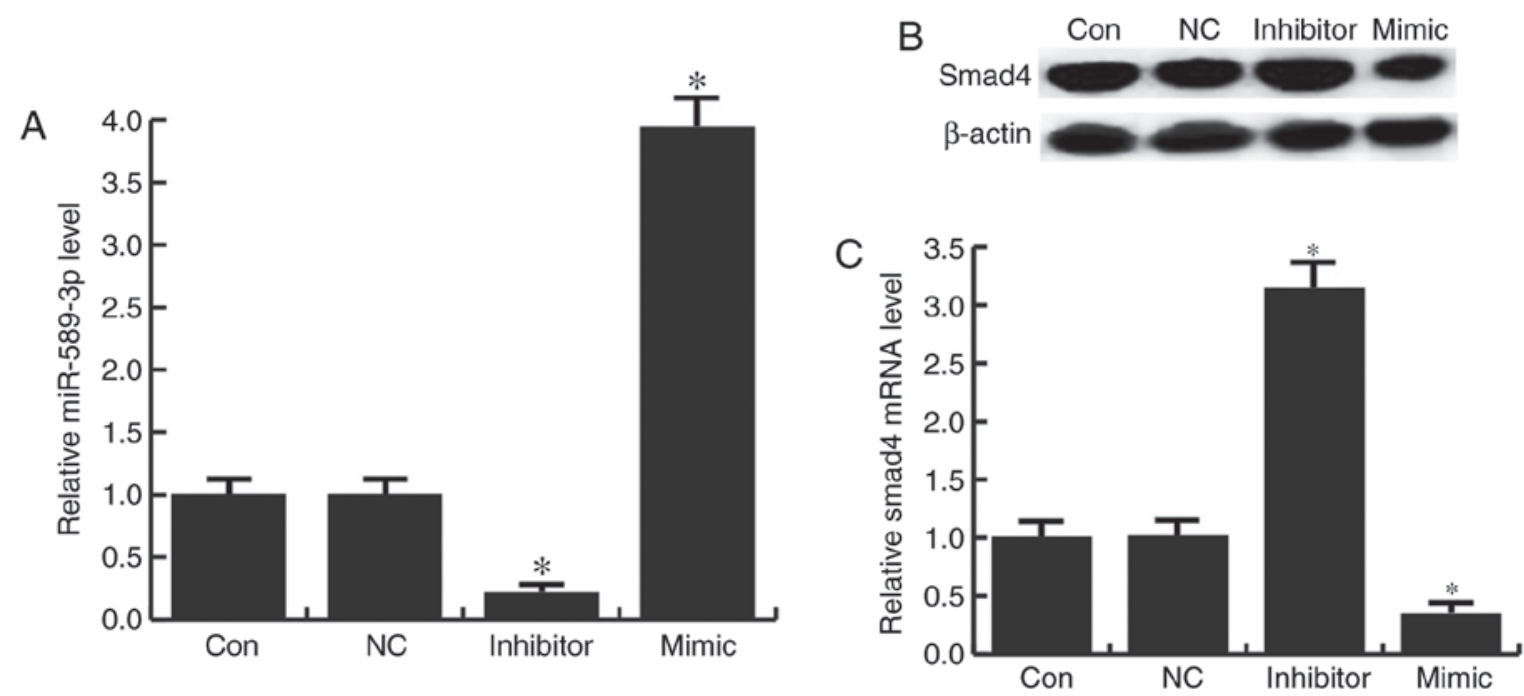

Figure 6. Smad4 is negatively regulated by miR-589-3p in NP cells. (A) miR-589-3p expression level in NP cells from different groups; (B) protein level of Smad4 in NP cells was detected by western blotting; (C) mRNA level of Smad4 in NP cells was detected by RT-qPCR. Con, normal NP cells; NC, NP cells transfected with NC oligonucleotides; mimic, NP cells transfected with miR-589-3p mimic (50 nM); inhibitor, NP cells transfected with miR-589-3p inhibitor $(100 \mathrm{nM}) .{ }^{*} \mathrm{P}<0.01$ vs. Con. Experiments were performed in triplicate.

To further confirm miR-589-3p regulating Smad4 expression in NP cells, we transfected NP cells with miR-589-3p mimics, miR-589-3p inhibitors, or its negative control respectively. RT-qPCR was performed to detect the transfection efficiency (Fig. 6A). miR-589-3p mimics significantly inhibited the mRNA and protein expression of SMAD4 in NP cells, while the miR-589-3p inhibitor increased SMAD4 mRNA and protein levels (Fig. 6B and C). These results indicate that miR-589-3p may function in LDD via regulating SMAD4.

\section{Discussion}

A growing number of studies have revealed that miRNAs play important roles in a variety of pathological processes, as well as in regulating cell proliferation, apoptosis and differentiation (18). Extensive research has proved the important roles of miRNAs in the development of IDD. For example, Hu et al reported that miR-194 and miR-515 can relieve the biosynthesis of chondroitin sulfate during human IDD development (19). Lv et al suggest that miR-146a over-expression can promote IDD through regulating TRAF/NF- $\mathrm{BB}$ pathway (20). miR-15 has been found upregulated in IDD and it can promote IDD by targeting MAP3K9 (21). miR-133a was downregulated in IDD and it involves in IDD progression via regulating Type II collagen by targeting MMP9 (22). A previous study suggested that miR-589 was over-expressed in IDD. To date, the role of miR-589-3p, a rarely studied miRNA, in IDD remain unknown. Therefore, this study we aimed to examined the expression of miR-589-3p in IDD, as well as to study the role of miR-589-3p in IDD and explore the underlying molecular mechanisms. We hope to find a novel and effective therapeutic target for the diagnosis and treatment of LDD.

The present study found that miR-589-3p was upregulated in human LDD. One of the major features of IDD is decrease of the proteoglycan (PG) content of IVDs (23). LPS, an admitted strong promoter of inflammation, can reduce the PG content, thus leading to IDD $(24,25)$. Thus, in the present study, we used LPS to establish the IDD cell model for further study of miR-589-3p, and we found miR-589-3p was upregulated in human NP cells by LPS stimulated.

Excessive apoptosis of IVD cells and undue degradation of ECM involve in the development of IDD $(26,27)$. Inflammation also plays an important role in disc degeneration. A variety of proinflammatory cytokines, including TNF- $\alpha$, IL-1 $\beta$, IL-6 and IL-12, were notably enhanced due to immunoreactivity in the generative IVD tissues (28). Our findings suggested that miR-589-3p inhibitors significantly reduced the production of TNF- $\alpha$, IL- $1 \beta$ and IL- 6 in LPS stimulated NP cells. Moreover, miR-589-3p inhibitors inhibited LPS stimulated NP cell apoptosis, and significantly enhanced type II collagen and aggrecan expression in LPS stimulated NP cells.

As a critical pro-inflammatory factor, TGF- $\beta$ has been reported could enhance catabolic genes expression and repress critical ECM genes expression through the NP cells and AF cells in intervertebral disc tissue (29). Smad4, one of thousands of the potential target genes of miR-589-3p, is a co-activator and mediator of TGF- $\beta$ signal pathway. Thus, we choose Smad4 for further investigation, and our results showed that miR-589-3p directly targets Smad4, and negatively regulate Smad4 expression in NP cells. The result was consistent with the previous reports (16). Therefore, these results suggested that miRNA-589-3p may affect the ECM and inflammation by targeting Smad4.

Taken together, our results indicated facilitate effect of miR-589-3p on IDD progress. This is the first study clarifying the underlying mechanisms of miR-589-3p in IDD. miR-589-3p may serve as a novel therapeutic target for LDD.

\section{Acknowledgements}

The present study was supported by the Science and Technology Support Program of Zhangjiagang (development of society) (no. ZKS1412), and the Special Clinical Treatment of Key Diseases of Suzhou City (no. LCZX201416). 


\section{References}

1. Yang X, Kong Q, Song Y, Liu L, Zeng J and Xing R: The characteristics of spinopelvic sagittal alignment in patients with lumbar disc degenerative diseases. Eur Spine J 23: 569-575, 2014.

2. Mayer JE, Iatridis JC, Chan D, Qureshi SA, Gottesman O and Hecht AC: Genetic polymorphisms associated with intervertebral disc degeneration. Spine J 13: 299-317, 2013.

3. Adams MA and Roughley PJ: What is intervertebral disc degeneration, and what causes it? Spine (Phila Pa 1976) 31: 2151-2161, 2006

4. Freemont AJ: The cellular pathobiology of the degenerate intervertebral disc and discogenic back pain. Rheumatology (Oxford) 48: 5-10, 2009.

5. Li Z, Shen J, Wu WK, Yu X, Liang J, Qiu G and Liu J: The role of leptin on the organization and expression of cytoskeleton elements in nucleus pulposus cells. J Orthop Res 31: 847-857, 2013.

6. Li Z, Liang J, Wu WK, Yu X, Yu J, Weng X and Shen J: Leptin activates RhoA/ROCK pathway to induce cytoskeleton remodeling in nucleus pulposus cells. Int J Mol Sci 15: 1176-1188, 2014

7. Bartel DP: MicroRNAs: Genomics, biogenesis, mechanism, and function. Cell 116: 281-297, 2004.

8. Lee RC, Feinbaum RL and Ambros V: The C. elegans heterochronic gene lin-4 encodes small RNAs with antisense complementarity to lin-14. Cell 75: 843-854, 1993.

9. Croce CM: Causes and consequences of microRNA dysregulation in cancer. Nat Rev Genet 10: 704-714, 2009.

10. Teague EM, Print CG and Hull ML: The role of microRNAs in endometriosis and associated reproductive conditions. Hum Reprod Update 16: 142-165, 2010.

11. Yu X, Li Z, Shen J, Wu WK, Liang J, Weng X and Qiu G: MicroRNA-10b promotes nucleus pulposus cell proliferation through RhoC-Akt pathway by targeting HOXD10 in intervetebral disc degeneration. PLoS One 8: e83080, 2013.

12. Ohrt-Nissen S, Dessing KB, Rossing M, Lajer C, Vikeså J, Nielsen FC, Friis-Hansen L and Dahl B: Characterization of miRNA expression in human degenerative lumbar disks. Connect Tissue Res 54: 197-203, 2013.

13. Liu G, Cao P, Chen H, Yuan W, Wang J and Tang X: MiR-27a regulates apoptosis in nucleus pulposus cells by targeting PI3K. PLoS One 8: e75251, 2013.

14. Liu H, Huang X, Liu X, Xiao S, Zhang Y, Xiang T, Shen X, Wang $G$ and Sheng B: miR-21 promotes human nucleus pulposus cell proliferation through PTEN/AKT signaling. Int J Mol Sci 15: 4007-4018, 2014.

15. Wang HQ, Yu XD, Liu ZH, Cheng X, Samartzis D, Jia LT, Wu SX, Huang J, Chen J and Luo ZJ: Deregulated miR-155 promotes Fas-mediated apoptosis in human intervertebral disc degeneration by targeting FADD and caspase-3. J Pathol 225 : 232-242, 2011
16. Hu P, Feng B, Wang G, Ning B and Jia T: Microarray based analysis of gene regulation by microRNA in intervertebral disc degeneration. Mol Med Rep 12: 4925-4930, 2015.

17. Zhao L, Lu X and Cao Y: MicroRNA and signal transduction pathways in tumor radiation response. Cell Signal 25: 1625-1634, 2013.

18. Ell B and Kang Y: MicroRNAs as regulators of bone homeostasis and bone metastasis. Bonekey Rep 3: 549, 2014.

19. Hu B, Xu C, Tian Y, Shi C, Zhang Y, Deng L, Zhou H, Cao P, Chen $\mathrm{H}$ and Yuan W: Inflammatory microRNA-194 and -515 attenuate the biosynthesis of chondroitin sulfate during human intervertebral disc degeneration. Oncotarget 8: 49303-49317, 2017.

20. Lv F, Huang Y, Lv W, Yang L, Li F, Fan J and Sun J: MicroRNA-146a ameliorates inflammation via TRAF6/NF- $\kappa B$ pathway in intervertebral disc cells. Med Sci Monit 23: 659-664, 2017.

21. Cai P, Yang T, Jiang X, Zheng M, Xu G and Xia J: Role of miR-15a in intervertebral disc degeneration through targeting MAP3K9. Biomed Pharmacother 87: 568-574, 2017.

22. Xu YQ, Zhang ZH, Zheng YF and Feng SQ: Dysregulated miR-133a mediates loss of type II collagen by directly targeting matrix metalloproteinase 9 (MMP9) in human intervertebral disc degeneration. Spine (Phila Pa 1976) 41: 717-724, 2016.

23. Ngo K, Patil P, McGowan SJ, Niedernhofer LJ, Robbins PD, Kang J, Sowa G and Vo N: Senescent intervertebral disc cells exhibit perturbed matrix homeostasis phenotype. Mech Ageing Dev 166: 16-23, 2017.

24. Ellman MB, Kim JS, An HS, Chen D, KC R, An J, Dittakavi T, van Wijnen AJ, Cs-Szabo G, Li X, et al: Toll-like receptor adaptor signaling molecule MyD88 on intervertebral disk homeostasis: In vitro, ex vivo studies. Gene 505: 283-290, 2012.

25. Iwata M, Ochi H, Asou Y, Haro H, Aikawa T, Harada Y, Nezu Y, Yogo T, Tagawa M and Hara Y: Variations in gene and protein expression in canine chondrodystrophic nucleus pulposus cells following long-term three-dimensional culture. PLoS One 8: e63120, 2013 .

26. Zhao CQ, Jiang LS and Dai LY: Programmed cell death in intervertebral disc degeneration. Apoptosis 11: 2079-2088, 2006.

27. Kepler CK, Ponnappan RK, Tannoury CA, Risbud MV and Anderson DG: The molecular basis of intervertebral disc degeneration. Spine J 13: 318-330, 2013.

28. Shamji MF, Setton LA, Jarvis W, So S, Chen J, Jing L, Bullock R, Isaacs RE, Brown $\mathrm{C}$ and Richardson WJ: Proinflammatory cytokine expression profile in degenerated and herniated human intervertebral disc tissues. Arthritis Rheum 62: 1974-1982, 2010.

29. Risbud MV and Shapiro IM: Role of cytokines in intervertebral disc degeneration: Pain and disc content. Nat Rev Rheumatol 10: 44-56, 2014. 